\title{
Cocaine Regulates Endocannabinoids-Containing Extracellular Vesicles Release in Ventral Tegmental Area via Sigma-1 Receptor and ADP-Ribosylation Factor 6 Pathway
}

\author{
Yoki Nakamura ${ }^{1,2}$, Dilyan I Dryanovski ${ }^{3}$, Shelley N Jackson ${ }^{4}$, Shang-Yi A Tsai ${ }^{2}$, Amina S Woods ${ }^{4}$, \\ Carl R Lupica ${ }^{3}$, Tsung-Ping $\mathrm{Su}^{2}$ \\ ${ }^{I}$ Department of Pharmacology, Graduate School of Biomedical \& Health Sciences, Hiroshima University, Hiroshima, \\ Japan, ${ }^{2}$ Cellular Pathobiology Section, Integrative Neuroscience Research Branch, Intramural Research Program, \\ National Institute on Drug Abuse, Baltimore, MD, USA, ${ }^{3}$ Electrophysiology Research Section, Cellular Neurobiology \\ Research Branch, Intramural Research Program, National Institute on Drug Abuse, Baltimore, MD, USA, ${ }^{4}$ Structural \\ Biology Unit, Intramural Research Program, National Institute on Drug Abuse, Baltimore, MD, USA
}

Background: Cocaine activates dopamine neurons in the ventral tegmental area by mobilizing endocannabinoid 2arachidonoylglycerol (2-AG) to attenuate the inhibitory tone of the gamma-aminobutyric acid. However, the exact underlying mechanisms of cocaine caused 2-AG release remain unknown. Here we found that 2-AG exists in the extracellular vesicle $(\mathrm{EV})$ and that cocaine increases the release of EV via the pluripotent modulator in living systems the sigma-1 receptor (Sig1R).

Methods: The EV samples were typically prepared from the midbrain slices of 2 wild type male C57BL/6J mice by ultracentrifugation and filtration $(1 \mu \mathrm{m})$. Drugs were injected i.p. at a volume of $5 \mathrm{ml} / \mathrm{kg}$. Thirty and $60 \mathrm{~min}$ after i.p. injections with cocaine $(15 \mathrm{mg} / \mathrm{kg})$, midbrain slices were collected. Injections of Sig1R antagonist BD1063 (10 mg/kg, s. c.), ADP-ribosylation factor 6 (ARF6) GEF inhibitor SecinH3 $(10 \mu \mathrm{mol} / \mathrm{kg}$, s.c.), or vehicle (s.c.) were performed $20 \mathrm{~min}$ prior to injections of saline or cocaine. 2-AG in the EV samples were measured by mass spectrometry analyses.

Results: We examined the EV levels by the quantification of the EV markers including integrin $\beta$ 1, Alix, and ARF6, as well as the dopamine (DA) neuronal marker tyrosine hydroxylase (TH). Those EV markers were detected in the EV sample collected from the mouse midbrain. Interestingly, EVs derived from midbrain, but not cortex or hippocampus, contained TH. Thus, those data suggest that DA neurons in the mouse midbrain region can release TH-containing EVs into extracellular space. Moreover, $30 \mathrm{~min}$, but not $60 \mathrm{~min}$, after an injection of cocaine, a significant elevation of extracellular $\mathrm{TH}$ in the form of EV was observed in the mouse midbrain. This effect of cocaine was blocked by pretreatment with either BD1063 or SecinH3. In addition, we found that the EV samples contain 2-AG.

Conclusions: Taken together, our results suggest that cocaine regulates the release of 2-AG containing EV from mouse DA neurons via the new Sig1R-ARF6 pathway described in this report. (supported by IRP NIDA NIH) 